\title{
On the Normal Boundary Intersection Method for Generation of Efficient Front
}

\author{
Pradyumn Kumar Shukla \\ Institute of Numerical Mathematics \\ Department of Mathematics \\ Dresden University of Technology Dresden PIN 01069, Germany \\ pradyumn.shukla@mailbox.tu-dresden.de
}

\begin{abstract}
This paper is concerned with the problem of finding a representative sample of Pareto-optimal points in multi-objective optimization. The Normal Boundary Intersection algorithm is a scalarization scheme for generating a set of evenly spaced Efficient solutions. A drawback of this algorithm is that Pareto-optimality of solutions is not guaranteed. The contributions of this paper are two-fold. First, it presents alternate formulation of this algorithms, such that (weak) Pareto-optimality of solutions is guaranteed. This improvement makes these algorithm theoretically equivalent to other classical algorithms (like weighted-sum or $\varepsilon$-constraint methods), without losing its ability to generate a set of evenly spaced Efficient solutions. Second, an algorithm is presented so as to know beforehand about certain sub-problems whose solutions are not Pareto-optimal and thus not wasting computational effort to solve them. The relationship of the new algorithm with weighted-sum and goal programming method is also presented.
\end{abstract}

Keywords: Multi-objective optimization, Efficient front generation, Computationally efficient algorithm.

\section{Introduction}

There are usually multiple conflicting objectives in engineering problems (see for example [512:13:311]). Since the objectives are in conflict with each other there is a set of Pareto-optimal solutions. The main goal of multi-objective optimization is to seek Pareto-optimal solutions. Over the years there have been various approaches toward fulfillment of this goal. Usually it is not economical to generate the entire Pareto-surface, due to the high computational cost for function evaluations in engineering problems one aims for finding a representative sample of Pareto-optimal points. It have been observed that convergence and diversity are two conflicting criterion's which are desired in any algorithm which tries to generate the entire efficient front. There are many algorithms for generating the Pareto-surface for general non-convex multi-objective problems. See for example 4,103] and the references therein.

Most classical generating multi-objective optimization methods use an iterative scalarization scheme of standard procedures such as weighted-sum or 
$\varepsilon$-constraint method as discussed in 410, the drawbacks of most of these approaches is that although there are results for convergence, diversity is hard to maintain. Thus we see that systematic variation of parameters in these scalarization techniques do not guarantee diversity in the solution sets as discussed in ([1]). The Normal Boundary Intersection (NBI) algorithm [2] gave a breakthrough by using scalarizing schemes that give a good diversity in the objective space. It start from equidistant points on the utopia plane (plane passing through individual function minimizers) and then go along a certain direction. The progress along the direction is measured by an auxiliary real variable. There are also some other algorithms also which try to generate evenly spaced Efficient solutions. Weck [7] developed adaptive weighted sum method for multi-objective optimization. Messac 9 developed the Normal Constraint method for getting even representation of the efficient frontier.

The developments in this paper are aimed at developing an improved algorithm which like direction-based algorithms produces evenly spaced points on the Efficient front and on the other hand like weighted-sum or $\varepsilon$-constraint method does not produce dominated points. This paper also proposes a way to jump over some of non-convex regions in which no Pareto-optimal solution lies. This amounts to solving a smaller number of sub-problems compared to existing methods.

The rest of the paper is structured as follows: in Section 2, the NBI algorithm is briefly described and an improved algorithm is also presented. A method to jump over dominated regions in non-convex cases is also presented. Section 3 discusses the relationship of the new algorithm with the weighted-sum and goal programming method. Finally conclusions are presented in the last section.

\section{NBI Algorithm: Original and a New Formulation}

The NBI method was developed by Das et. al. 2] for finding a uniformly spread Pareto-optimal solutions for a general nonlinear multi-objective optimization problem. The weighted-sum scalarization approach has a fundamental drawback of not being able to find a uniform spread of Pareto-optimal solutions, even if a uniform spread of weight vectors are used. The NBI approach uses a scalarization scheme with a property that a uniform spread in parameters will give rise to a near uniform spread in points on the efficient frontier. Also, the method is independent of the relative scales of different objective functions. The scalarization scheme is briefly described below.

Let us consider the following multi-objective problem (MP):

$$
\begin{array}{ll}
\min _{\mathbf{x} \in S} & F(\mathbf{x}), \\
\text { where } & S=\{\mathbf{x} \mid h(\mathbf{x})=0 ; g(\mathbf{x}) \leq 0, a \leq \mathbf{x} \leq b\}
\end{array}
$$

Let $F^{*}=\left(f_{1}^{*}, f_{2}^{*}, \ldots, f_{m}^{*}\right)^{\top}$ be the ideal point of the multi-objective optimization problem with $m$ objective functions and $n$ variables. Henceforth we shift the origin (in objective space) to $F^{*}$ so that all the objective functions are nonnegative. Let the individual minimum of the functions be attained at $\mathbf{x}_{i}^{*}$ for each $i=1,2, \ldots, m$. The convex hull of the individual minima is then obtained. 
The simplex obtained by the convex hull of the individual minimum can be expressed as $\Phi \beta$, where $\Phi=\left(F\left(\mathbf{x}_{1}^{*}\right), F\left(\mathbf{x}_{2}^{*}\right), \ldots F\left(\mathbf{x}_{m}^{*}\right)\right)$ is a $m \times m$ matrix and $\beta=\left\{\left(b_{1}, b_{2}, \ldots, b_{m}\right)^{\top} \mid \sum_{i=1}^{m} b_{i}=1\right\}$. The original study suggested a systematic method of setting $\beta$ vectors in order to find a uniformly distributed set of efficient points. The NBI scalarization scheme takes a point on the simplex and then searches for the maximum distance along the normal pointing toward the origin. The NBI subproblem $\left(\mathrm{NBI}_{\beta}\right)$ for a given vector $\beta$ is as follows:

$$
\begin{aligned}
\max _{(\mathbf{x}, t)} & t, \\
\operatorname{subject} & \Phi \beta+t \hat{n}=F(\mathbf{x}), \\
& \mathbf{x} \in S,
\end{aligned}
$$

where $\hat{n}$ is the normal direction at the point $\Phi \beta$ pointing towards the origin. The solution of the above problem gives the maximum $t$ and also the corresponding Pareto-optimal solution, $\mathbf{x}$. The method works even when the normal direction is not an exact one, but a quasi-normal direction. The following quasi-normal direction vector is suggested in Das et al. [2]: $\hat{n}=-\Phi e$, where $e=(1,1, \ldots, 1)^{T}$ is a $m \times 1$ vector. The above quasi-normal direction has the property that $\mathrm{NBI}_{\beta}$ is independent of the relative scales of the objective functions.

The main problem with NBI method is that the solutions of the sub-problems need not be Pareto-optimal (not even locally). This method aims at getting boundary points rather than Pareto-optimal points. Pareto-optimal points are a subset of boundary points. These obtained point may or may not be a Paretooptimal point. Figure 1 shows the obtained solutions (i.e. $a, b, c, d, e, f, g, h$ ) corresponding to equidistant points (i.e. $A, B, C, D, E, F, G, H$ ) on the convex hull (i.e. line $A H$ ).

It can be seen that points $d, e$ and $f$ are not Pareto-optimal but are still found using the NBI method. It is because solutions of NBI sub-problem is only constrained to lie on a line, however for any point $\mathbf{x}^{*}$, to be Pareto-optimal, the existence of any point in the objective space which satisfies $F(x) \leq F\left(\mathbf{x}^{*}\right)$ need to be checked (since such a point dominates $F\left(\mathrm{x}^{*}\right)$ ).

Using the concept of the goal attaintment approach of Gembicki [6] we formulate a modified algorithm (called as mNBI) in which we solve the following sub-problem $\left(\mathrm{mNBI}_{\beta}\right)$ for a given vector $\beta$ is as follows:

$$
\begin{aligned}
& \max _{(\mathbf{x}, t)} \quad t, \\
& \operatorname{subject} \text { to } \Phi+t \hat{n} \geq F(\mathbf{x}), \\
& \mathbf{x} \in S,
\end{aligned}
$$

This change in formulation increases the feasible space so as to check for points which dominate the points on the line. Figure 2 shows the schematics. It can be seen that the points $d, e$ and $f$ which are not Pareto-optimal will not be found using the mNBI method. Points $x, y$ and $z$ dominate the corresponding points $d, e$ and $f$ (which results in larger value of $t$ ) and they are thus not optimal to $m N B I_{\beta}$. For the optimality of $\mathrm{mNBI}_{\beta}$ subproblems we have the following result. 

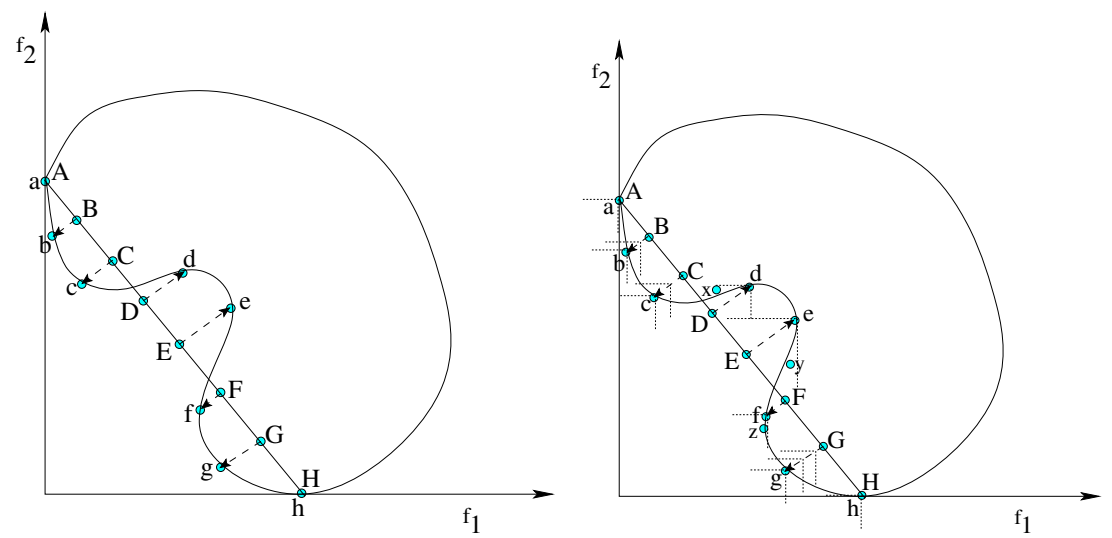

Fig. 1. Schematic showing obtained so- Fig. 2. Schematic showing obtained solutions using NBI method lutions using a modified NBI method

Lemma 1. Let $\left(t^{*}, x^{*}\right)$ be the optimal solution of (3) subproblem then $x^{*}$ is weakly efficient.

Proof: The proof follows from [6] by using $\Phi \beta$ and $t \hat{n}$ as the goal vector and direction respectively.

Another advantage of the mNBI algorithm is that since for non-convex problems all boundary points need not be Pareto-optimal, for some non-convex regions we can ignore (jump over the non-efficient regions in the objective space) solving certain sub-problems. Figure 3 shows the sketch of our proposed method to this. Suppose we start $\mathrm{mNBI}_{\beta}$ from the individual minima $A$ of $f_{1}$ and move on the line $A B$ (convex hull of individual minima). When we reach point $C$ the optimal solution of mNBI gives the (weakly) efficient point $x$. Note that the optimal solution of the NBI subproblem would have been $y$. From the constraints in mNBI subproblem we can calculate whether or not the constraints are active at optima. For example in this case the constraints $\Phi \beta+t \hat{n} \geq F(\mathbf{x})$ are not-active since the optima $x$ does not lies on the line. Now since the obtained solution $x$ will always weakly dominate the region $x+\mathbb{R}_{+}^{m}$ (Lemma 1). Since we know the direction $\hat{n}$ (constant for all sub-problems) we an easily find the point $D$ on the line $A B$ (convex hull of individual minima, in general a simplex), which corresponds to obtained solution $x$.

Lemma 2. Let $\left(t^{*}, x^{*}\right)$ be the optimal solution of (3). Let $w:=\Phi \beta+t^{*} \hat{n}$. Let $\pi[S] \subseteq \mathbb{R}^{m-1}$ denote the projection (in the direction $\hat{n}$ ) of any surface $S \subseteq \mathbb{R}^{m}$ onto the simplex obtained by convex hull of individual minima. Then the solution of (3) for all $\beta \in \pi\left[\left(F(x)+\mathbb{R}_{+}^{m}\right) \bigcap\left(w-\mathbb{R}_{+}^{m}\right)\right]$ is not Pareto-optimal.

Proof: The proof follows by noting that $x$ weakly dominates the region $(F(x)+$ $\left.\mathbb{R}_{+}^{m}\right)$ and that there exist no feasible point in $\left(w-\mathbb{R}_{+}^{m}\right)$ that dominates $w$. 


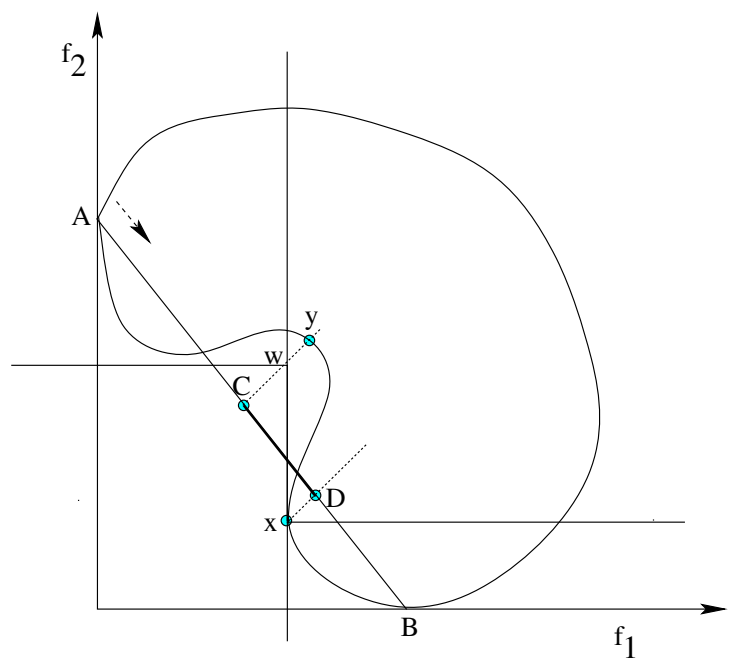

Fig. 3. Schematic showing how to avoid solving certain sub-problems whose solutions are not Pareto-optimal

For our example, all subproblems originating from region $C D$ do not give Paretooptimal solutions and thus we can ignore them. It is to be noted that this information is obtained before solving the sub-problems. This amounts to solving a smaller number of sub-problems compared to existing methods. This computational gain can be of significant interest in problems where the cost of function evaluation is high.

\section{Relationship Between mNBI Subproblem and Weighted Sum Method}

Given a Pareto-optimal solution $\mathbf{x}^{*}$, let $\bar{h}(\mathbf{x})$ denote the augmented vector of all constraints that are active at $\mathbf{x}^{*}$. We can think of the problem to be constrained only by $\bar{h}(\mathbf{x})$. Let $w \in \mathbb{R}^{m}, \sum_{i=1}^{m} w_{i}=1$, denote positive weights for the objectives. We consider the weighted linear combination problem as follows

$$
\begin{aligned}
& \max _{(\mathbf{x})} w^{\top} F(\mathbf{x}), \\
& \text { subject to } \bar{h}(\mathbf{x})=0 .
\end{aligned}
$$

The problem (4) ) will be denoted by $L C_{w}$. Part of first-order necessary KKT conditions for optimality of $\left(\mathrm{x}^{*}, \lambda^{*}\right)$ for $L C_{w}$ is

$$
\nabla_{\mathbf{X}} F\left(\mathbf{x}^{*}\right) w+\nabla_{\mathbf{x}} \bar{h}\left(\mathbf{x}^{*}\right) \lambda^{*}=0 .
$$

In a similar way, the $\mathrm{mNBI}_{\beta}$ subproblem can be written as

$$
\begin{array}{ll}
\min _{(\mathbf{x}, t)} & -t, \\
\text { subject to } & F(\mathbf{x})-\Phi \beta-t \hat{n} \leq 0, \\
& \bar{h}(\mathbf{x})=0,
\end{array}
$$


Part of first-order KKT condition for optimality of $\left(\mathbf{x}^{*}, t^{*}, \lambda^{(1) *}, \lambda^{(2) *}\right)$ is

$$
\begin{aligned}
& \nabla_{\mathbf{X}} F\left(\mathbf{x}^{*}\right) \lambda^{(1) *}+\nabla_{\mathbf{X}} \bar{h}\left(\mathbf{x}^{*}\right) \lambda^{(2) *}=0 \\
& -1-\hat{n} \lambda^{(1) *}=0
\end{aligned}
$$

where $\lambda^{(1) *} \in \mathbb{R}^{m}, \lambda^{(1) *} \geq 0$ represents the multipliers corresponds to the inequality constraints $F(\mathbf{x})-\Phi \beta-t \hat{n} \leq 0$, and $\lambda^{(2) *}$ denote the multipliers of the equality constraints $\bar{h}(\mathbf{x})=0$.

Claim. Suppose $\left(\mathbf{x}^{*}, t^{*}, \lambda^{(1) *}, \lambda^{(2) *}\right)$ is the solution of $\mathrm{mNBI}_{\beta}$ and $\sum_{i=1}^{m} \lambda_{i}^{(1) *} \neq 0$. Then $L C_{w}$ with the weight vector $w=\frac{1}{\sum_{i=1}^{m} \lambda_{i}^{(1) *}} \lambda^{(1) *}$ has the solution

$$
\left[\mathbf{x}, \lambda^{*}=\frac{1}{\sum_{i=1}^{m} \lambda_{i}^{(1) *}} \lambda^{(2) *}\right]
$$

Proof: Dividing both sides of first part of (17) by the positive scalar $\sum_{i=1}^{m} \lambda_{i}^{(1) *}$ and observing that $\bar{h}(\mathbf{x})=0$, the equivalence follows.

Hence we can obtain the weighting of objectives that correspond to solution of $\mathrm{mNBI}_{\beta}$ subproblem. Note that if one uses the original NBI method to get the equivalence, since the $F(\mathbf{x})-\Phi \beta-t \hat{n}=0$ are equality constraints the components of multiplier $\lambda^{(1) *} \in \mathbb{R}^{m}$ can be positive or negative. Thus one could obtain negative weights [2]. In such a case the solution of $\mathrm{NBI}_{\beta}$ subproblem is non Pareto-optimal. This is because irrespective of whether the solution lies in a convex or a non-convex part of the boundary, it should satisfy the first-order necessary KKT conditions if it is Pareto-optimal. However, this does not occurs in $\mathrm{mNBI}_{\beta}$ subproblems (the weights are always non-negative).

\section{Relationship Between mNBI Subproblem and Goal Programming}

A solution to an mNBI subproblem is also a solution to a goal programming problem.

Claim. Suppose $\left(\mathbf{x}^{*}, t^{*}, \lambda^{(1) *}, \lambda^{(2) *}\right)$ is the solution of $\mathrm{mNBI}_{\beta}$ and $\sum_{i=1}^{m} \lambda_{i}^{(1) *} \neq 0$. If $\lambda_{k}^{(1) *}$ is any strict positive component (note that all the components are nonnegative), then $\mathbf{x}^{*}$ solves the following goal programming problem:

$$
\begin{array}{ll}
\min _{(\mathbf{x})} & f_{k}(\mathbf{x}), \\
\text { subject to } & f_{i}(\mathbf{x}) \leq \gamma_{i}, \forall i \neq k, \\
& \bar{h}(\mathbf{x})=0,
\end{array}
$$

with for all $i \neq k$ the goals given by

$$
\gamma_{i}=\left\{\begin{array}{cc}
f_{i}(\mathbf{x}) & \text { if } \lambda_{i}^{(1) *} \neq 0 \\
\text { any finite number } \geq f_{i}(\mathbf{x}) & \text { if } \lambda_{i}^{(1) *}=0
\end{array}\right.
$$


Proof: Since $\left(\mathbf{x}^{*}, t^{*}, \lambda^{(1) *}, \lambda^{(2) *}\right)$ is the solution of $\mathrm{mNBI}_{\beta}$ it satisfies the firstorder necessary KKT condition (17). Dividing both sides of (7) by $\lambda_{k}^{(1) *}>0$ we obtain

$$
\nabla_{\mathbf{x}} f_{k}\left(\mathbf{x}^{*}\right) w+\sum_{i=1, i \neq k}^{i=n} \nabla_{\mathbf{x}} f_{i}\left(\mathbf{x}^{*}\right) \frac{\lambda_{i}^{(1) *}}{\lambda_{k}^{(1) *}}+\nabla_{\mathbf{x}} \bar{h}\left(\mathbf{x}^{*}\right) \frac{\lambda^{(2) *}}{\lambda_{k}^{(1) *}}=0 .
$$

Now with $\frac{\lambda_{i}^{(1) *}}{\lambda_{k}^{(1) *}} \geq 0$ as the multipliers of the $m-1$ inequality constraints in (8), the goals $\gamma_{i}$ satisfy complementarity by definition since

$$
\begin{aligned}
& \gamma_{i}=f_{i}(\mathbf{x}) \text { whenever } \lambda_{i}^{(1)} \neq 0, \\
& \frac{\lambda_{i}^{(1) *}}{\lambda_{k}^{(1) *}}\left(f_{i}(\mathbf{x})-\gamma_{i}\right)=0, \forall i \neq k .
\end{aligned}
$$

Using the above together with feasibility of $\mathbf{x}$ for (8), we obtain that the point $\left(\mathbf{x}^{*}, \frac{\lambda_{i}^{(1) *}}{\lambda_{k}^{(1) *}}, \frac{\lambda^{(2) *}}{\lambda_{k}^{(1) *}}\right)$ satisfies the first order necessary optimality conditions of (8)).

Note that as in the case of weighted-sum method if one uses the original NBI method to get the equivalence, due to the presence of equality constraints $F(\mathbf{x})-$ $\Phi \beta-t \hat{n}=0$, the components of multiplier $\lambda^{(1) *} \in \mathbb{R}^{m}$ can be positive or negative. Thus one could obtain negative weights. In such a case the equivalence of NBI and goal programming problem requires additional assumption that the components of $\lambda^{(1) *}$ are of the same sign while no such assumptions are needed in the mNBI method.

\section{Conclusions}

In this paper, we presented an efficient formulation of the NBI algorithm for getting an even spread of efficient points. This method unlike NBI method does not produce dominated points and is theoretically equivalent to weighted-sum or $\varepsilon$-constraint method This paper also proposes a way to reduce the number of subproblems to be solved for non-convex problems. We compare the mNBI method with other popular methods like weighted-sum method and goal programming methods using Lagrange multipliers. It turned out that mNBI method does not require any unusual assumption compared to relationship of NBI method with weighted-sum method and goal programming method. Lastly we would like to mention that since some other class of methods like the Normal-Constraint Method [9] or the Adaptive Weighted Sum Method 8] use similar line or inclined search based constraint in their sub-problems, the solutions of the sub-problems of these method are also in general not Pareto-optimal and hence the mNBI method presented in this paper is superior to them. 


\section{Acknowledgements}

The author acknowledges the partial financial support by the Gottlieb-Daimlerand Karl Benz-Foundation.

\section{References}

1. I. Das and J.E. Dennis. A closer look at drawbacks of minimizing weighted sum of objecties for Pareto set generation in multicriteria optimization problems. Structural Optimization, 14(1):63-69, 1997.

2. I. Das and J.E. Dennis. Normal-boundary intersection: A new method for generating the Pareto surface in nonlinear multicriteria optimization problems. SIAM Journal of Optimization, 8(3):631-657, 1998.

3. K. Deb. Multi-objective optimization using evolutionary algorithms. Chichester, UK: Wiley, 2001.

4. M. Ehrgott. Multicriteria Optimization. Berlin: Springer, 2000.

5. H. Eschenauer, J. Koski, and A. Osyczka. Multicriteria Design Optimization. Berlin: Springer-Verlag, 1990.

6. F. W. Gembicki. Performance and Sensitivity Optimization: A Vector Index Approach. PhD thesis, Case Western Reserve University, Cleveland, OH, 1974.

7. I. Y. Kim and O.de Weck. Multiobjective optimization via the adaptive weighted sum method. In 10th AIAA/ISSMO Multidisciplinary Analysis and Optimization Conference, 2004.

8. I. Y. Kim and O.de Weck. Adaptive weighted sum method for multiobjective optimization: a new method for Pareto front generation. Structural and Multidisciplinary Optimization, 31(2):105-116, 2005.

9. A. Messac and C. A. Mattson. Normal constraint method with guarantee of even representation of complete pareto frontier. AIAA Journal, 42(10):2101-2111, 2004.

10. K. Miettinen. Nonlinear Multiobjective Optimization. Kluwer, Boston, 1999.

11. Andrzej Osyczka. Evolutionary Algorithms for Single and Multicriteria Design Optimization. Physica Verlag, Germany, 2002. ISBN 3-7908-1418-0.

12. Eric Sandgren. Multicriteria design optimization by goal programming. In Hojjat Adeli, editor, Advances in Design Optimization, chapter 23, pages 225-265. Chapman \& Hall, London, 1994.

13. R. B. Stanikov and J. B. Matusov. Multicriteria Optimization and Engineering. New York: Chapman and Hall, 1995. 